\title{
Generation mean analysis of grain quality traits in selected rice populations derived from different amylose characteristics
}

\begin{abstract}
BACKGROUND: Genetic analysis using generation mean analysis is a tool for designing the most appropriate breeding approaches to developing varieties of rice. It estimates the gene actions that control quantitative traits, as well as the additive, dominance and epistatic effects. This study was conducted using three rice populations that were derived from parental lines with different amylose content. The aim was to partition the gene actions using generation mean analysis for the selected populations. RESULTS: A scaling test was carried out to evaluate the fulfilment of the additive-dominance model. Non-allelic interaction was observed for milled grain length, length-to-width ratio and milled rice recovery of all populations evaluated. An additive-dominance model was not adequate for amylose, gel consistency, grain length, grain width, milled grain width and head rice recovery, thus epistasis was involved in the populations evaluated. The importance of additive gene action was observed for grain length, milled grain length and milled rice recovery for populations of high- and low-amylose parents. However, populations with intermediate- and high-amylose parents and intermediate- and low-amylose parents shared almost similar dominance gene actions for most of the physical grain quality traits. CONCLUSION: These results suggested that delayed selection is the best approach for traits governed by dominance and epistasis effects. Meanwhile, the traits that were governed by additive effects should undergo thorough selection at an early stage.
\end{abstract}

Keyword: Additive-dominance model; Generation means; Grain quality traits; Rice variety 